\title{
Determination of the anisotropy fields of polycrystalline hexaferrites by FMR
}

\author{
Victor A. Zhuravlev", Alexander V. Zhuravlev, Vasily V. Atamasov, and Grigory I. Malenko \\ National Research Tomsk State University, 634050, Lenina av., 36, Tomsk, Russia
}

\begin{abstract}
We propose the method for calculating the ferromagnetic resonance curves of polycrystalline hexaferrites with anisotropy of the easy magnetization plane. The results of the experimental studies of the magnetocrystalline anisotropy fields and the magnetomechanical ratios of hexaferrites $\mathrm{Ba}_{3} \mathrm{Co}_{1.5+x} \mathrm{Ti}_{x} \mathrm{Fe}_{24.5-2 x} \mathrm{O}_{41}(0.0 \leq x \leq 1.0)$ and $\mathrm{Ba}_{3} \mathrm{Co}_{2.5-x} \mathrm{Zn}_{x} \mathrm{Ti}_{0.5}$ $\mathrm{Fe}_{23} \mathrm{O}_{41}(0.0 \leq x \leq 1.2)$ systems at room temperature are presented.
\end{abstract}

\section{Introduction}

Hexagonal oxide ferrimagnets (hexaferrites) are promising for use in various fields of modern technology. According to [1], there is a significant increase in the number of publications on the physical properties and various fields of hexaferrites application currently. Hexaferrites with a negative first anisotropy constant have a magnetic order such as easy magnetization plane (EMP). They have high values of magnetic permeability in the microwave range [2].

For the targeted use of hexaferrites, it is necessary to know the magnitude and sign of the magnetocrystalline anisotropy (MCA) fields $\left(H_{\mathrm{a}}\right)$, as well as the saturation magnetization values $\left(M_{\mathrm{S}}\right)$. If there are monocrystalline samples of hexaferrite the measurement of these parameters by FMR is not a problem [2-4]. However, the ferrimagnetic materials are usually made and used in the form of polycrystals or powders. Such materials are macroscopically isotropic. However, the FMR method allows one to determine the magnitudes of the MCA fields and the value of the effective magnetomechanical ratio $\gamma=g e / 2 m c$ of this materials. Here $g$ is the effective g-factor of the material under study, $e$ is the electron charge, $m$ is the electron mass, $c$ is the speed of light $[5,6]$.

A typical representative of hexaferrites with EMP is hexaferrite $\mathrm{Ba}_{3} \mathrm{Co}_{2} \mathrm{Fe}_{24} \mathrm{O}_{41}\left(\mathrm{Co}_{2} \mathrm{Z}\right)$ [2]. In our study, the following tasks were set: a) to propose a method for measuring the magnitudes of the magnetomechanical ratios and the anisotropy fields of hexaferrites with EMP from FMR; b) experimentally investigate the effect of substitution by the $\mathrm{Co}^{2+} \mathrm{Ti}^{4+}$ complex for $\mathrm{Fe}^{3+}$ ions in $\mathrm{Ba}_{3} \mathrm{Co}_{1.5+x} \mathrm{Ti}_{x} \mathrm{Fe}_{24.5-2 x} \mathrm{O}_{41} \quad(0.0 \leq x \leq 1.0)$ hexaferrite and substitution of $\mathrm{Co}^{2+}$ ions by non-magnetic $\mathrm{Zn}^{2+}$ ions in $\mathrm{Ba}_{3} \mathrm{Co}_{2.5-x} \mathrm{Zn}_{x} \mathrm{Ti}_{0.5} \mathrm{Fe}_{23} \mathrm{O}_{41}(0.0 \leq x$ $\leq 1.2$ ) hexaferrite on the magnetjcristalline anisotropy of these compounds.

* Corresponding author: ptica@mail.tsu.ru 


\section{Ferromagnetic resonance in polycrystalline hexaferrites with anisotropy of the easy magnetization plane}

For polycrystalline hexaferrites with the large values of the MCA fields, the independed grain approach is well performed. The fundamentals of the FMR theory in the approximation of non-interacting grains were developed by E. Schlömann for materials with cubic [7, 8] and hexagonal [9] crystal structures. In [10, 11], the theory was generalized to the case of polycrystalline hexaferrites with anisotropy of the magnetomechanical ratio.

The starting point of this approach is the calculation of the FMR line of a monocrystaline single-domain grain in the form of an ellipsoid of revolution with an arbitrary orientation of the magnetizing field $\left(\vec{H}_{0}\right)$ relative to the crystallographic axes of the grain. Further, by averaging over all possible orientations, the FMR curve of the polycrystal is calculated.

We assume that the sample consists of randomly oriented identical particles in the form of an ellipsoid of revolution with a hexagonal MCA. The density of the magnetic part of the free energy $(U)$ consists of contributions from the Zeeman energy $\left(U_{\text {Zee }}\right)$, the energy of demagnetizing fields $\left(U_{\mathrm{M}}\right)$, and the energy of magnetocrystalline anisotropy $\left(U_{\mathrm{MCA}}\right)$. In a spherical coordinate system, the free energy density $U=U_{\text {Zee }}+U_{\mathrm{M}}+U_{\mathrm{MCA}}$ is written:

$$
\begin{aligned}
& U=-M_{\mathrm{S}} H[\sin \Theta \sin \vartheta \cos (\Phi-\varphi)+\cos \Theta \cos \vartheta]+2 \pi M_{S}^{2}\left(N_{\perp} \sin ^{2} \vartheta+N_{\|} \cos ^{2} \vartheta\right)+ \\
& +k_{1} \sin ^{2} \vartheta+k_{2} \sin ^{4} \vartheta+k_{3} \sin ^{6} \vartheta+k_{4} \sin ^{6} \vartheta \cos 6 \varphi .
\end{aligned}
$$

Here $H$ is the external magnetizing field; $\Theta, \Phi$ - are polar and azimuthal angles of $H$; $\vartheta, \varphi-$ are polar and azimuthal angles of the magnetization vector; $k_{\mathrm{i}}-$ are the MCA constants, $N_{\perp}, N_{\|}$are the demagnetizing factors of the particle, $2 N_{\perp}+N_{\|}=1$. The anisotropy constant $k_{1}$ describes anisotropy relative to the hexagonal axis, the constants $k_{1}$, $k_{2}, k_{3}$ describe anisotropy relative to the basal plane, and the constant $k_{4}$ describes anisotropy in the basal plane of a single crystal grain.

The angles of equilibrium orientation of the magnetization vector $\left(\vartheta_{0}, \varphi_{0}\right)$ and the magnitude of the resonance field $H_{0}(\Theta, \Phi)$ are calculated from the solution of the system of three transcendental equations. The first two equations determine the equilibrium orientation of the magnetization vector, and the third equation is the Smith-Suhl resonance equation:

$$
\left\{\begin{array}{l}
\partial U /\left.\partial \vartheta\right|_{\substack{\vartheta=\vartheta_{0} \\
\varphi=\varphi_{0}}}=H\left[\sin \vartheta_{0} \cos \Theta-\cos \vartheta_{0} \sin \Theta \cos \left(\varphi_{0}-\Phi\right)\right]+ \\
+0.5 \sin 2 \vartheta_{0}\left[H_{a 1}^{\prime}+H_{a 2} \sin ^{2} \vartheta_{0}+\left(H_{a 3}+(1 / 6) H_{\Phi} \cos 6 \varphi_{0}\right) \sin ^{4} \vartheta_{0}\right]=0 ; \\
\partial U /\left.\partial \varphi\right|_{\substack{\vartheta=\vartheta_{0} \\
\varphi=\varphi_{0}}}=H \sin \vartheta_{0} \sin \Theta \sin \left(\varphi_{0}-\Phi\right)-(1 / 6) H_{\Phi} \sin ^{6} \vartheta_{0} \sin 6 \varphi_{0}=0 ; \\
{[f /(\gamma / 2 \pi)]^{2}-H_{1} H_{2}+H_{3}^{2}=0 .}
\end{array}\right.
$$

Here $H_{\mathrm{ai}}=2 i k_{\mathrm{i}} / M_{\mathrm{S}}(i=1,2,3), H_{\Phi}=36 k_{4} / M_{\mathrm{S}}, H_{\mathrm{a} 1}^{\prime}=H_{\mathrm{a} 1}-4 \pi M_{\mathrm{S}}\left(N_{\|}-N_{\perp}\right)$ are the anisotropy fields, $f$ is the frequency of the electromagnetic field, $\gamma$ is the magnetomechanical ratio. The values of $H_{1}, H_{2}, H_{3}$ are equal to:

$$
\begin{aligned}
& H_{1}=H\left[\sin \vartheta_{0} \sin \Theta \cos \left(\varphi_{0}-\Phi\right)+\cos \vartheta_{0} \cos \Theta\right]+H_{a 1}^{\prime} \cos 2 \vartheta_{0}+ \\
& +H_{a 2} \sin ^{2} \vartheta_{0}\left(4 \cos ^{2} \vartheta_{0}-1\right)+\left(H_{a 3}+(1 / 6) H_{\Phi} \cos 6 \varphi_{0}\right) \sin ^{4} \vartheta_{0}\left(6 \cos ^{2} \vartheta_{0}-1\right) \\
& H_{2}=H\left(\sin \Theta / \sin \vartheta_{0}\right) \cos \left(\varphi_{0}-\Phi\right)-H_{\Phi} \sin ^{4} \vartheta_{0} \cos 6 \varphi_{0} \\
& H_{3}=H \cos \vartheta_{0}\left(\sin \Theta / \sin \vartheta_{0}\right) \sin \left(\varphi_{0}-\Phi\right)-H_{\Phi} \sin ^{4} \vartheta_{0} \cos \vartheta_{0} \sin 6 \varphi_{0} .
\end{aligned}
$$


The system of equations (2) is solved numerically by changing the angles of the magnetizing field in the ranges $90^{\circ} \geq \Theta \geq 1^{\circ}, 0^{\circ} \leq \Phi \leq 30^{\circ}$. The ranges of angles are determined by the symmetry of the energy of the MCA. The practice of calculating resonance curves $[10,11]$ has shown that it is enough to change the angles in increments of $1^{\circ}$. As a result of the calculations, we obtain a matrix of equilibrium angles $\vartheta_{0}(\Theta, \Phi), \varphi_{0}(\Theta, \Phi)$ and resonance fields $H_{0}(\Theta, \Phi)$ for a given grid of angles of the magnetizing field. The value of the resonance field for the values $\Theta=0^{\circ}, 0^{\circ} \leq \Phi \leq 30^{\circ}$ is:

$$
H_{0}\left(0^{\circ}, \Phi\right)=f /(\gamma / 2 \pi)-H_{a 1}^{\prime}
$$

The imaginary part of the diagonal component of the permeability tensor of singlecrystal grains is calculated by the formula [12]:

$$
\mu^{\prime \prime}(H, \Theta, \Phi)=\frac{\alpha\left(4 \pi M_{\mathrm{S}}\right) H_{0}(\Theta, \Phi)\left[H^{2}+\left(1+\alpha^{2}\right) H_{0}^{2}(\Theta, \Phi)\right]}{\left[H^{2}-\left(1+\alpha^{2}\right) H_{0}^{2}(\Theta, \Phi)\right]^{2}+\left[2 \alpha H H_{0}(\Theta, \Phi)\right]^{2}} .
$$

Here, $\alpha$ is the damping constant in the Landau - Lifshitz - Hilbert equation of an individual grain. The imaginary part of the magnetic permeability of a polycrystalline or powder sample is calculated by averaging (5) over the grid of angles $\Theta$, $\Phi$ by the formula:

$$
\mu_{p o l i}^{\prime \prime}(H)=(3 / 2 \pi) \int_{0}^{\pi / 2} \int_{0}^{\pi / 6} \mu^{\prime \prime}(H, \Theta, \Phi) \sin \Theta d \Theta d \Phi .
$$

The results of calculating the resonance curves using formulas (2) - (6) are shown in Figures 1 and 2. The FMR curves in Fig. 1 are calculated without taking into account anisotropy in the basal plane $\left(H_{\Phi}=0 \mathrm{kOe}\right)$. The resonanse curves in Fig. 2 are calculated for three values of $H_{\Phi}=0.5,1.0$, and $2.0 \mathrm{kOe}$. The calculations were performed for the parameters: $f=36 \mathrm{GHz}, M_{\mathrm{S}}=270 \mathrm{Gs}, \gamma / 2 \pi=2.8 \mathrm{GHz} / \mathrm{kOe}$ and the damping constant $\alpha=$ 0.07 . The values of $M_{\mathrm{S}}$ and $\alpha$ are characteristic of the materials for which we presented the experimental data below. The vertical lines in Fig. 1 and 2 correspond to resonance fields calculated by formulas (4) and (7):

$$
\begin{aligned}
& {[f /(\gamma / 2 \pi)]^{2}=\left[H_{0}\left(90^{\circ}, 0^{\circ}\right)+H_{\Phi}\right]\left[H_{0}\left(90^{\circ}, 0^{\circ}\right)-H_{\Theta}+H_{\Phi} / 6\right] ;} \\
& {[f /(\gamma / 2 \pi)]^{2}=\left[H_{0}\left(90^{\circ}, 30^{\circ}\right)-H_{\Phi}\right]\left[H_{0}\left(90^{\circ}, 30^{\circ}\right)-H_{\Theta}-H_{\Phi} / 6\right] .}
\end{aligned}
$$

Here $H_{\Theta}=H_{\mathrm{a} 1}^{\prime}+H_{a 2}+H_{a 3}$ is the anisotropy field relative to the basal plane.

According to Fig. 1, the presence of a high-field feature near the field $H_{0}\left(0^{\circ}, \Phi\right)$ on the resonance curves makes it possible to determine the anisotropy field $H_{a 1}^{\prime}$ using formula (4). The anisotropy field $H_{\Theta}$ can be estimated using formulas (7) at $H_{\Phi}=0$. Thus, if in an experiment on resonance curves two features are observed in fields close to $H_{0}\left(90^{\circ}, 0^{\circ}\right)$ and $H_{0}\left(0^{\circ}, \Phi\right)$, the contribution from the higher-order anisotropy fields can be distinguished by the formula $H_{a 2}+H_{a 3}=H_{\Theta}-H_{\mathrm{a} 1}^{\prime}$.

Figure 2 illustrates the effect of the anisotropy field $H_{\Phi}$ on the shape of the resonance curve. It is seen that an estimate of the magnitude of this field from experiment can be obtained only for materials with $H_{\Phi} \geq 1 \mathrm{kOe}$. We did not estimate the value of $H_{\Phi}$ from the experiment because the opposite inequality holds for the materials studied by us.

Figure 3 shows the results of comparing the parameters of the resonance curves of the polycrystals: the maxima (M) on the FMR curves and the maxima of the derivatives (MD) with the calculation of the FMR frequencies of a single crystal (line) using formulas (4), (7) at $H_{\Phi}=0$. It is seen that for polycrystals with EMP for the low-field singularity the best agreement with (7) is obtained for the average value of the fields corresponding to the maxima of the derivatives and the maxima of the FMR curves $-\left(H_{\mathrm{MD}}+H_{\mathrm{M}}\right) / 2$, and for the 
high-field singularity - with the maximum of the derivative $H_{\mathrm{MD}}$. In this regard, the processing of the experimental FMR spectra in order to determine the anisotropy fields is carried out as follows.

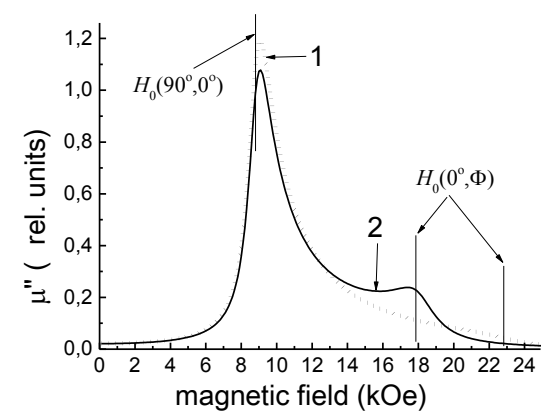

Fig. 1. Resonance curves of polycrystalline hexaferrite with EMP. Curve 1 (points): anisotropy fields $\boldsymbol{H}_{\mathrm{a} 1}=-10 \mathrm{kOe}, \boldsymbol{H}_{\mathrm{a} 2}=\boldsymbol{H}_{\mathrm{a} 3}=$ $\boldsymbol{H}_{\boldsymbol{\Phi}}=\mathbf{0}$ kOe. Curve 2 (line): anisotropy fields $H_{\mathrm{a} 1}=-5 \mathrm{kOe}, \boldsymbol{H}_{\mathrm{a} 2}=-5 \mathrm{kOe}, \boldsymbol{H}_{\mathrm{a} 3}=\boldsymbol{H}_{\Phi}=0 \mathrm{kOe}$.

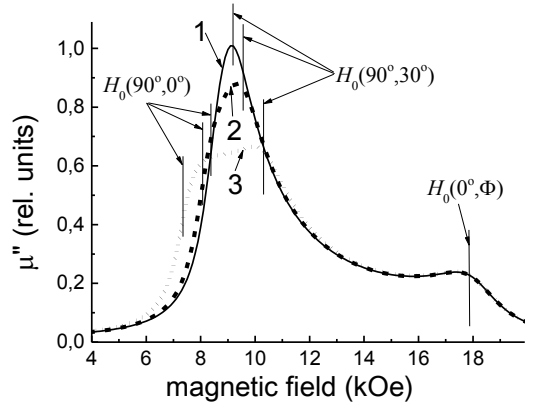

Fig. 2. Resonance curves of polycrystalline hexaferrite with EMP. Curve 1: $\boldsymbol{H}_{\mathrm{a} 1}=-5 \mathrm{kOe}$, $H_{\mathrm{a} 2}=-5 \mathrm{kOe}, H_{\mathrm{a} 3}=0 \mathrm{kOe}, H_{\Phi}=0.5 \mathrm{kOe}$. Curve 2: $\boldsymbol{H}_{\mathrm{a} 1}=-\mathbf{5} \mathrm{kOe}, \boldsymbol{H}_{\mathrm{a} 2}=-\mathbf{5} \mathrm{kOe}, \boldsymbol{H}_{\mathrm{a} 3}=\mathbf{0} \mathrm{kOe}$, $\boldsymbol{H}_{\Phi}=\mathbf{1}$ kOe. Curve 3: $\boldsymbol{H}_{\mathrm{a} 1}=-\mathbf{5} \mathrm{kOe}, \boldsymbol{H}_{\mathrm{a} 2}=-\mathbf{5}$ $\mathrm{kOe}, \boldsymbol{H}_{\mathbf{a} 3}=\mathbf{0} \mathrm{kOe}, \boldsymbol{H}_{\mathbf{\Phi}}=\mathbf{2} \mathrm{kOe}$.

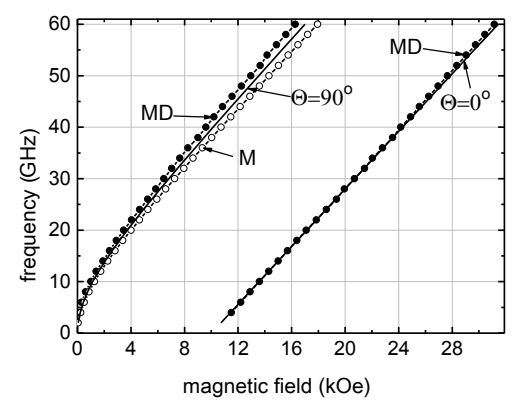

Fig. 3. FMR frequencies for a material with anisotropy of the EMP type, $H_{\mathrm{a} 1}=-10 \mathrm{kOe}, H_{\mathrm{a} 2}=$ $H_{\mathrm{a} 3}=H_{\Phi}=0 \mathrm{kOe}, \alpha=0.1, \gamma / 2 \pi=2.8 \mathrm{GHz} /$ kOe.

1. FMR curves are measured in the frequency range, which depends on the magnitude of the anisotropy fields.

2. The dependences of the resonant frequencies on the magnetizing fields corresponding to the average value $\left(H_{\mathrm{MD}}+H_{\mathrm{M}}\right) / 2$ (low field maximum) and the maximum of derivatives (high field maximum) are plotted. By processing these dependences using the least squares method, the values of $\gamma / 2 \pi$ and the approximate values of the anisotropy fields, are estimated using the formulas (7) and (4).

3. By a detailed comparison of the shapes of the calculated and experimental FMR curves, the magnitude of the magnetomechanical relations and the anisotropy fields are refined.

\subsection{MCA fields of the hexaferrites system $\mathrm{Ba}_{3} \mathrm{Co}_{1.5+x} \mathrm{Ti}_{x} \mathrm{Fe}_{24.5-2 x} \mathrm{O}_{41}$}

The measured by FMR magnetomechanical ratios and anisotropy fields of hexaferrite system $\mathrm{Ba}_{3} \mathrm{Co}_{1.5+x} \mathrm{Ti}_{x} \mathrm{Fe}_{24.5-2 x} \mathrm{O}_{41}(0.0 \leq x \leq 1.0)$ are presented in Table 1 . 
Table 1. Magnetomechanical ratios and MCA fields of the $\mathrm{Ba}_{3} \mathrm{Co}_{1.5+x} \mathrm{Ti}_{x} \mathrm{Fe}_{24.5-2 x} \mathrm{O}_{41}(0.0 \leq x \leq 1.0)$.

$\begin{array}{llllllllll}\text { Concentration, } x & 0.0 & 0.2 & 0.3 & 0.4 & 0.6 & 0.7 & 0.8 & 0.9 & 1.0 \\ \gamma / 2 \pi, \text { GHz/kOe } & 2.81 & 2.83 & 2.84 & 2.83 & 2.84 & 2.83 & 2.84 & 2.84 & 2.84 \\ H_{\Theta}, \text { kOe } & -10.3 & -11.0 & -11.8 & -11.8 & -12.1 & -12.5 & -12.5 & -13.0 & -14.0 \\ H_{\text {a1 }}, \text { kOe } & -6.5 & -8.7 & -9.5 & -9.8 & - & - & - & - & - \\ H_{\text {a2 }}, \text { kOe } & -2.5 & -2.3 & -2.3 & -2.0 & - & - & - & - & -\end{array}$

According to Table 2, the magnitude of the magnetomechanical ratios within the experimental error $( \pm 0.02 \mathrm{GHz} / \mathrm{kOe})$ does not change with $x$. With an increase in the concentration of ions of the $\mathrm{Co}^{2+} \mathrm{Ti}^{4+}$ complex, the anisotropy fields $H_{\mathrm{a} 1}, H_{\Theta}$ are increased. Hexaferrite with $x=0.5$ is not included in this table, since this composition is the initial for the second hexaferrite system that we studied.

\subsection{MCA fields of the hexaferrites system $\mathrm{Ba}_{3} \mathrm{Co}_{2.5-x} \mathrm{Zn}_{x} \mathrm{Ti}_{0.5} \mathrm{Fe}_{23} \mathrm{O}_{41}$}

The measured by FMR magnetomechanical ratios and anisotropy fields of hexaferrite system системы $\mathrm{Ba}_{3} \mathrm{Co}_{2.5-x} \mathrm{Zn}_{x} \mathrm{Ti}_{0.5} \mathrm{Fe}_{23} \mathrm{O}_{41}(0.0 \leq x \leq 1.2)$ are presented in Table 2 .

Table 2. Magnetomechanical ratios and MCA fields of the $\mathrm{Ba}_{3} \mathrm{Co}_{2.5-x} \mathrm{Zn}_{x} \mathrm{Ti}_{0.5} \mathrm{Fe}_{23} \mathrm{O}_{41}(0.0 \leq x \leq 1.2)$

$\begin{array}{llllllllllll}\text { Concentration, } & 0.0 & 0.1 & 0.2 & 0.3 & 0.4 & 0.5 & 0.6 & 0.7 & 0.8 & 1.0 & 1.2 \\ \boldsymbol{x} & & & & & & & & & & & \\ \gamma / 2 \pi, \mathrm{GHz} / \mathrm{kOe} & 2.83 & 2.83 & 2.83 & 2.83 & 2.82 & 2.82 & 2.83 & 2.83 & 2.82 & 2.83 & 2.83 \\ H_{\Theta}, \text { kOe } & -11.5 & -11.0 & -11.1 & -11.0 & -10.0 & -9.5 & -10.5 & -9.5 & -9.5 & -7.5 & -6.7 \\ M_{S}, \text { Gs } & 247 & 253 & 257 & 262 & 266 & 270 & 274 & 277 & 281 & 288 & 293\end{array}$

According to Table 2, the magnetomechanical ratios within the measurement error are independent of the replacement of cobalt ions by zinc ions. The value of the anisotropy field $H_{\Theta}$ decreases with increasing $x$. The magnitude of the saturation magnetization increases with increasing $x$.

\section{Conclusion}

Thus, a method for estimating the magnitudes of the fields of magnetocrystalline anisotropy and the magnetomechanical ratio from studies of ferromagnetic resonance in polycrystalline hexaferrites with anisotropy of the easy magnetization plane is proposed.

It is shown that the substitution of ferric ions by the $\mathrm{Co}^{2+} \mathrm{Ti}^{4+}$ complex leads to an increase in the anisotropy field relative to the basal plane $\left(H_{\Theta}\right)$.

Substitution of $\mathrm{Co}^{2+}$ ions by nonmagnetic $\mathrm{Zn}^{2+}$ ions in $\mathrm{Ba}_{3} \mathrm{Co}_{2.5} \mathrm{Ti}_{0.5} \mathrm{Fe}_{23} \mathrm{O}_{41}$ hexaferrite decreases the value of the $H_{\Theta}$ anisotropy field, but leads to an increase in the saturation magnetization.

This work was performed under the Program for Improving Tomsk State University Competitiveness among the World's Leading Scientific and Educational Centers. Measurements were carried out on the equipment of the Tomsk Regional Common Use Center: Center of radio-physics measurements, diagnostic and researching of parameters of natural and artificial materials [14].

\section{References}

1. R.C. Pullar, Prog. Mat. Sci. 57, 1191 (2012)

2. J. Smit, H.P.J. Wijn, Ferrites. Physical properties of ferrimagnetic oxides in relation to their technical applications (Eindhoven: Philips Technical Library, 1959) 
3. A.A. Oshlakov, V.A. Zhuravlev, Russian Physics Journal, 43, 804 (2000)

4. V.A. Zhuravlev, E.P. Naiden, A.A. Oshlakov, Russian Physics Journal, 44, 806 (2001)

5. V.A. Zhuravlev, V.I. Itin, R.V. Minin, Yu. M. Lopushnyak, V. A. Svetlichnyi, I. N. Lapin, D. A. Velikanov, I. Yu. Lilenko, J. All. Comp. 771, 686 (2019)

6. V.A. Zhuravlev, E.P. Naiden, Phys. of the Solid State. 51, 327 (2009)

7. E. Schlömann, J. Phys. Chem. Solids, 6, 257 (1958)

8. E. Schlömann, J. Phys. Radium, 20, 327(1959)

9. E. Schlömann, R.V. Jones, J. Appl. Phys., 30, 177 (1959)

10. V.A. Zhuravlev, Physics of the Solid State, 41, 956 (1999)

11. V. A. Zhuravlev, V. A. Meshcheryakov, Russ. Phys. J., 56, No. 12, 1387 (2014)

12. A. G. Gurevich, G. A. Melkov, Magnetization oscillations and Waves (CRC Press. 1996)

13. V.Yu. Kreslin, E.P. Naiden, Instruments and Experimental Techniques, 4555 (2002)

14. The electronic resource: http://www.ckp.tsu.ru/ckp_3/ 